\title{
ESTIMULAÇÃO ELÉTRICA NERVOSA TRANSCUTÂNEA (TENS) NO PÓS-OPERATÓRIO DE CESARIANA
}

\author{
Melo de Paula G ${ }^{1}$, Molinero de Paula VR ${ }^{1}$, Dias RO ${ }^{2}$, Mattei K ${ }^{2}$ \\ ${ }^{1}$ Departamento de Fisioterapia, Universidade de Rio Verde, Rio Verde, GO \\ ${ }^{2}$ Fisioterapeuta \\ Correspondência para: Gustavo Melo de Paula, Rua Avelino Faria 477, apto 904, Centro, CEP 75900-000, Rio Verde, GO
}

Recebido: 23/05/2005 - Aceito: 16/11/2005

\begin{abstract}
RESUMO
Objetivo: Verificar o efeito da aplicação da TENS no quadro álgico de pacientes submetidas à cirurgia de cesariana. Método: Foi utilizada uma amostragem de 30 mulheres, voluntárias, com faixa etária entre 16 e 35 anos, em período imediato de pósoperatório de cesariana, com a presença de dor abdominal e no baixo ventre, subdivididas em dois grupos A e B, contendo em cada um deles 15 mulheres, submetidas, respectivamente, à eletroestimulação e ao tratamento placebo por eletroestimulação. A rotina hospitalar de administração de fármacos não foi alterada e manteve-se similar em ambos os grupos. A corrente utilizada foi a TENS convencional $(\mathrm{F}=100 \mathrm{~Hz}$ e $\mathrm{T}=50 \mu \mathrm{s})$, com pulso bipolar assimétrico, que foi aplicada através de quatro eletrodos de borracha siliconada e carbonada, gel e fita adesiva para a fixação dos mesmos de forma peri-incisional em técnica bipolar com dois pares de eletrodos cruzados, após cessar o efeito da anestesia, por 50 minutos, com intensidade em um nível sensorial forte. A Escala Visual Analógica (EVA) foi utilizada para a quantificação da intensidade da dor antes e após a eletroestimulação. Para a análise dos dados foi aplicado o teste $t$ de Student, para amostras pareadas, com um nível de significância de 5\%. Resultados: Foi demonstrada uma diferença estatisticamente significante entre as intensidades da dor, antes e após a aplicação da TENS, apenas nos indivíduos participantes do grupo A. Conclusão: Levando em consideração as condições experimentais deste estudo, podemos concluir que a aplicação da TENS pode constituir mais um recurso usado em mulheres submetidas à cesariana.
\end{abstract}

Palavras-chave: TENS, eletroestimulação, cesariana.

\section{ABSTRACT \\ Transcutaneous Electrical Nerve Stimulation (TENS) Following Cesarean Surgery}

Objective: To investigate the effect of transcutaneous electrical nerve stimulation (TENS) on pain in patients who underwent cesarean surgery. Method: A sample of 30 female volunteers aged between 16 and 35 years old was recruited. All were in the immediate postoperative period following cesarean surgery, presenting abdominal and lower-womb pain. They were divided into two groups of 15 women (A and B). Group A was treated with electrostimulation and Group B received placebo treatment. The hospital's drug administration routine was not altered and remained similar in the two groups. Conventional TENS current ( $\mathrm{F}=100 \mathrm{~Hz}$ and $\mathrm{T}=50 \mu \mathrm{s}$ ) with an asymmetrical bipolar pulse was applied through four siliconized and carbonized rubber electrodes, using gel and adhesive tape to attach these electrodes around the incision. The application was bipolar, using two pairs of crossed electrodes, at a high sensory level for 50 minutes, after interrupting the effect of the anesthesia. A visual analog scale was used for quantifying pain intensity before and after electrostimulation. For the data analysis, Student's t test for paired samples was utilized with a significance level of 5\%. Results: There was a statistically significant difference between the pain intensities before and after TENS application, for the individuals in group A alone. Conclusion: For the experimental conditions in this study, it can be concluded that TENS application may constitute an additional resource for use on women who have undergone cesarean surgery.

Key words: TENS, electrostimulation, cesarean. 


\section{INTRODUÇÃO}

A utilização de correntes elétricas terapêuticas constitui um dos vários recursos utilizados na fisioterapia. Uma vez moduladas com parâmetros apropriados, estas correntes podem atuar em diferentes condições, tais como: promover analgesia, contrações musculares, melhoria do fluxo circulatório local, drenagem de líquidos, tonificação ou relaxamento muscular, bem como incentivar a regeneração e a cicatrização de diversos tecidos corporais ${ }^{1}$.

Rushton ${ }^{2}$ relatou que uma das principais correntes elétricas terapêuticas, utilizada em processos álgicos agudos e crônicos, chamada TENS, cuja tradução em inglês referese a uma estimulação elétrica nervosa transcutânea, é usada com finalidade de influenciar e modular o processo de neurocondução da dor e atuar sobre a liberação de opióides endógenos a nível medular e da hipófise.

Este recurso tem sido usado extensivamente em locais de atendimento à saúde para o manejo sintomático de dor aguda e dor crônica de origem benigna e também no atendimento paliativo da dor causada por doenças ósseas metastáticas e neoplasias ${ }^{3,4,5}$.

A TENS constitui uma modalidade terapêutica não invasiva, de fácil manejo, que não apresenta efeitos colaterais ou interações com medicamentos, sendo utilizada para o alívio da dor pela estimulação de nervos periféricos, utilizando-se de eletrodos a nível da pele, estando baseada na liberação de opióides e na teoria do portão, ou também denominada teoria das comportas medulares de Melzack e Wall ${ }^{6}$.

Os autores anteriormente citados propuseram que a condução das informações de dor aguda e crônica, transmitidas, respectivamente, pelas fibras A-Delta e Tipo C, ambas de diâmetros estreitos, poderiam ser inibidas pela atividade de fibras aferentes periféricas do tipo A-Beta, de diâmetro largo, ou pela atividade de vias descendentes do cérebro relacionadas com a inibição da dor.

O estímulo nervoso transcutâneo inclui a transmissão de corrente elétrica através da pele, o qual vai atuar sobre os mecanoceptores periféricos, e, a partir daí, ser conduzido pela fibra A-Beta até um conjunto de interneurônios, que por sua vez atuam na inibição da retransmissão, a nível medular, dos estímulos dolorosos conduzidos pelas fibras A-Delta e Tipo $\mathrm{C}^{2,7}$.

Sendo assim, os efeitos analgésicos relacionam-se com um mecanismo de "fechamento da entrada" nas colunas dorsais da medula espinhal e pode estar associada também com a liberação de opióides endógenos, uma vez utilizandose um nível de intensidade forte de eletroestimulação, a qual vai induzir a liberação destas substâncias a nível encefálico e medular ${ }^{2,8}$.

Muito embora a TENS tenha sido estudada desde o final da década de 70, em condições inflamatórias, como método de analgesia na dor pós-operatória, em situações que envolveram a implantação de osteossínteses, em reconstruções musculares e tendinosas, bem como em cirurgias abdominais e pélvicas, trata-se de uma terapêutica ainda desconhecida por alguns profissionais da saúde e que apresenta algumas divergências de opinião em relação ao seu poder analgésico ${ }^{5}$, ${ }^{6}$.

Hollinger ${ }^{9}$ realizou um estudo que envolveu 72 mulheres submetidas à cirurgia de cesariana, sendo que 46 delas utilizaram a TENS convencional no período imediato de pósoperatório e as outras 26 não utilizaram. Foi verificado que não houve diferença entre os dois grupos no que diz respeito ao período de permanência hospitalar, no entanto, verificouse uma redução da administração de fármacos analgésicos no grupo experimental.

Smith et al. ${ }^{10}$ analisaram 18 mulheres no pós-parto de cesariana, divididas em dois grupos, submetidos à aplicação real e placebo da TENS. Os resultados demonstraram uma redução significativa da dor superficial e ao redor da sutura cirúrgica apenas no grupo experimental. No entanto, não verificaram diferença significativa na redução da dor intraabdominal, profunda, a qual os autores atribuem ao acúmulo de gases e à redução do peristaltismo dos órgãos e vísceras abdominais, que costuma ocorrer como resultado dos fármacos que são administrados durante a cirurgia.

Reynolds et al..$^{11}$ estudaram dois grupos de 25 mulheres, submetidas à cesariana, e verificaram que em ambos os grupos, sendo um deles submetido à aplicação da TENS convencional e o outro controle, não houve diferença significativa no período de permanência hospitalar e na quantidade de fármacos utilizados no pós-operatório. A quantidade, em média, de meperidina administrada via venosa no grupo experimental foi de 511mg em comparação com 456mg utilizada no grupo controle.

Lantsev e Smirnov ${ }^{12}$ analisaram 210 mulheres no pósparto imediato de cesariana, nas quais se aplicou a TENS convencional em 160 mulheres e as outras 50, utilizaram apenas medicação analgésica endovenosa. Foi verificado efeito analgésico significativo em ambos os grupos, com uma proporção de $77,5 \%$ de resultados bons e excelentes de analgesia nas pacientes do grupo submetido a TENS.

Navarro e Pacheco ${ }^{13}$ realizaram um estudo que envolveu 50 mulheres com dor pós-operatória de cesariana, divididas em dois grupos. O primeiro foi submetido à aplicação da TENS convencional, e no segundo, foi utilizado, para controle da dor, apenas 1 grama de dipirona via endovenosa, considerada pelos autores como dose terapêutica. Os resultados demonstraram que em ambos os grupos houve a necessidade de administração adicional de medicação analgésica, mas no grupo que usou a TENS, verificou-se uma maior redução da intensidade e do período de permanência da dor, bem como da quantidade de fármacos adicionalmente utilizados.

Lianza $^{5}$ relatou que a dor pós-operatória em região abdominal causa um grande desconforto e costuma retardar 
o retorno dos indivíduos às suas atividades de vida diária, e sendo assim, os mesmos costumam fazer uso de recursos farmacológicos e não farmacológicos apresentando cada um deles vantagens e desvantagens.

Segundo Polden e Mantle ${ }^{14}$ a TENS pode ser utilizada como recurso coadjuvante na situação específica do trabalho de parto, com o intuito de facilitar o processo de analgesia durante e no pós-parto e minimizar o desconforto e as complicações para os pacientes.

Ferreira e Payno ${ }^{15}$ relataram ainda que este recurso terapêutico se constitui num método simples, prático, de baixo custo e de resolução rápida, sem, no entanto, promover efeitos colaterais para os pacientes, podendo favorecer ainda a redução e ou até mesmo a eliminação da administração de fármacos no pós-parto.

Levando em consideração as vantagens inerentes a analgesia que pode ser produzida pela TENS, baseada na fisiologia da dor a partir da teoria do controle de comportas medulares e liberação de opióides endógenos, este estudo teve como objetivo verificar o efeito da aplicação real e placebo da TENS no quadro álgico de pacientes no pós-operatório de cesariana.

\section{CASUÍSTICA E MÉTODO}

Para a realização deste estudo foi utilizada uma amostragem total de 30 voluntárias, com faixa etária entre 16 e 35 anos, em período imediato de pós-operatório, cujos partos, do tipo cesariana, foram realizados na Maternidade Augusta Gomes Bastos, situada no município de Rio Verde-GO.

Todas as participantes foram esclarecidas quanto aos procedimentos experimentais do estudo e após terem concordado em participar, assinaram um termo de consentimento informado segundo resolução CNS 196/1996.

Como critério de inclusão a parturiente deveria apresentar uma intensidade de dor superior à zero na Escala Visual Analógica (EVA), com escala numérica respondida verbalmente (0-10) e ser primigesta ou ter sido submetida, no máximo, a dois partos anteriores, para que houvesse, realmente, uma situação de dor presente, pois segundo alguns autores ${ }^{16,17}$ a dor tende a ser minimizada e melhor suportada pelas pacientes, à medida que ocorre mais de dois partos, e isto poderia interferir nos resultados do estudo.

A amostragem foi subdividida, aleatoriamente, em dois grupos A e B. O grupo A constou de 15 mulheres, que foram submetidas à eletroestimulação por TENS, e o grupo B, também com 15 mulheres, foi submetido apenas a um tratamento placebo por eletroestimulação.

Antes da eletroestimulação dos indivíduos do grupo A, os mesmos elegiam um número representativo da intensidade de sua dor na EVA, com escala numérica verbal (0-10), e logo após, foram posicionados em decúbito dorsal no leito hospitalar, com exposição da área abdominal e de baixo ventre, para facilitar o posicionamento dos eletrodos e ser efetuada a aplicação da TENS.

Durante a realização da eletroestimulação, efetuada sempre por um único observador, foram utilizados 4 eletrodos de $15 \mathrm{~cm}^{2}(5 \mathrm{~cm} \mathrm{x} \mathrm{3cm)} \mathrm{de} \mathrm{borracha} \mathrm{siliconada} \mathrm{e} \mathrm{carbonada,}$ gel condutor e fita adesiva para fixá-los de forma periincisional em técnica bipolar cruzada na região de baixo ventre e um eletroestimulador KW modelo "Kinesis New Microcontrolled $^{\circledR}$ ", novo e devidamente calibrado pelo fabricante.

Foi utilizada a corrente TENS convencional $(\mathrm{F}=100 \mathrm{~Hz}$ e $\mathrm{T}=50 \mu \mathrm{s}$ ) com pulso bipolar assimétrico, aplicada após cessar o efeito da anestesia, por 50 minutos, sendo o cálculo feito pelo contador de tempo do aparelho, com intensidade modulada em um nível sensorial forte, a qual era modificada constantemente para a manutenção de uma sensação de formigamento intenso na região de baixo ventre das parturientes, as quais foram esclarecidas previamente sobre este processo.

Para os indivíduos do grupo $\mathrm{B}$, foram adotados os mesmos procedimentos, diferindo-se apenas no que diz respeito à regulagem da intensidade da corrente, ficando o aparelho desligado a fim de se proceder com um efeito placebo, sendo as parturientes esclarecidas que a sensação inerente à aplicação deste recurso seria a presença ou ausência de uma sensação de formigamento na região estimulada.

Decorridos 30 minutos após cessar a eletroestimulação com a TENS convencional no grupo A e TENS placebo no grupo $\mathrm{B}$, os pacientes novamente pontuavam a intensidade de suas dores na EVA, tendo como referência a intensidade inicialmente elegida por eles, que lhes eram repassadas pelo observador.

Os procedimentos de rotina adotados pela equipe de saúde, incluindo a aplicação endovenosa de cerca de 400mg de meperidina, mantiveram-se similares em ambos os grupos.

Com o objetivo de verificar a existência ou não de diferenças estatisticamente significantes entre as intensidades da dor inicial e após a aplicação de eletroestimulação nos indivíduos do grupo A e tratamento placebo nos indivíduos do grupo $\mathrm{B}$, foi aplicado o teste $t$ de Student com um nível de significância de 5\%.

\section{RESULTADOS}

A amostra constou de 30 mulheres em período imediato de pós-operatório de cesariana com idade entre 16 e 35 anos, no grupo A (média =2,7 e desvio-padrão (DP) =5,98 anos) e no grupo $\mathrm{B}$ (média $=23,13$ e $\mathrm{DP}=3,66$ anos), com peso entre 60 e 130 Kilogramas (Kg), no grupo A (média =71,23 e DP =18,86 Kg) e no grupo B (média =62,87 e DP =14,55 $\mathrm{Kg})$.

A identificação dos indivíduos participantes do estudo, a idade, o peso, o número de partos e o valor numérico representativo da intensidade da dor, inicialmente, e após a 
eletroestimulação e o tratamento placebo, respectivamente, nos grupos A e B, estão descritos nas tabelas 1 e 2. Para comparar as intensidades da dor no início e após a aplicação da TENS convencional e placebo nos grupos A e B, utilizou - se o teste $t$ de Student ( $\mathrm{p}<5 \%$ ), o qual permitiu-nos verificar uma redução significativa da intensidade da dor somente nos indivíduos do grupo A.

\section{DISCUSSÃO}

Muito embora alguns autores ${ }^{9,10}$ não tenham verificado efetividade da TENS na promoção de analgesia, em condições álgicas diversas, incluindo àquela presente no pós-operatório de cesariana, os nossos resultados corroboram com os de Lantsen e Smirnov ${ }^{12}$, Navarro e Pacheco ${ }^{13}$, Van de Spank ${ }^{18}$, Mendoza et al. ${ }^{19}$ e Van der Ploeg ${ }^{20}$ os quais verificaram a efetividade da TENS, em associação ou não, com outros recursos farmacológicos de analgesia, aplicados em situação de assistência obstétrica.

É bem interessante observar que neste estudo foi verificado, no grupo submetido à eletroestimulação placebo, uma regressão da dor em somente dois pacientes, a qual se atribui à meperidina introduzida via endovenosa, sendo que em dez pacientes, a intensidade da dor manteve-se constante, e em três delas, foi aumentada.

Já no grupo experimental, foi verificada uma regressão e, até mesmo, eliminação total da intensidade da dor, em quatorze mulheres, sendo que, em apenas uma delas, houve aumento da intensidade da dor, a qual nós acreditamos estar relacionada com uma ansiedade intensa apresentada por esta paciente, atribuída ao choro prolongado apresentado por ela, tanto no pré quanto no pós-parto, o que não foi verificado em nenhuma outra parturiente.

Na modulação dos parâmetros da corrente utilizada neste estudo, utilizamos uma modalidade de TENS convencional, com parâmetros de freqüência alta, baixa largura de pulso e intensidade forte, para permitir uma analgesia rápida e mais efetiva nas dores agudas das nossas voluntárias, conforme descrito e aplicado em condições semelhantes às nossas por Lantsen e Smirnov ${ }^{12}$ e Navarro e Pacheco ${ }^{13}$.

Em relação à colocação dos eletrodos, seguimos a metodologia de Navarro e Pacheco ${ }^{13}$ e os preceitos de Lianza ${ }^{5}$, o qual considera que a aplicação de uma corrente elétrica analgésica, com 4 eletrodos cruzados e dispersos nos dermátomos de T11 e T12, situados na região de baixo ventre, é mais efetiva para reduzir um quadro de dor presente nesta região.

Vale ressaltar que para o manejo e a quantificação da dor das pacientes que participaram deste estudo foram utilizados recursos simples, sem custos adicionais, efetivos e de fácil reprodutibilidade, tal qual a TENS, que se mostrou bem efetiva no alívio da dor pós-operatória de cesariana, sem apresentar efeitos colaterais ou interação com os medicamentos administrados, e, a EVA verbal, com valores

Tabela 1. Dados relativos aos indivíduos do grupo A (tratado).

\begin{tabular}{|c|c|c|c|c|c|}
\hline Identificação & Idade (anos) & Peso (Kg) & Partos & EVA inicial & $\begin{array}{c}\text { EVA após a } \\
\text { TENS }\end{array}$ \\
\hline 01 & 20 & 79 & 2 & 5 & 0 \\
\hline 02 & 18 & 58 & 1 & 3 & 0 \\
\hline 03 & 34 & 59 & 2 & 5 & 0 \\
\hline 04 & 20 & 68 & 2 & 5 & 2 \\
\hline 05 & 25 & 67 & 2 & 8 & 5 \\
\hline 06 & 27 & 95 & 2 & 9 & 8 \\
\hline 07 & 31 & 65,5 & 2 & 7 & 0 \\
\hline 08 & 20 & 58 & 2 & 10 & 2 \\
\hline 09 & 16 & 60 & 2 & 5 & 0 \\
\hline 10 & 19 & 130 & 2 & 7 & 0 \\
\hline 11 & 26 & 68 & 2 & 5 & 10 \\
\hline 12 & 17 & 64 & 1 & 9 & 0 \\
\hline 13 & 27 & 72 & 2 & 10 & 0 \\
\hline 14 & 28 & 62 & 2 & 9 & 0 \\
\hline 15 & 18 & 63 & 1 & 8 & 0 \\
\hline
\end{tabular}


Tabela 2. Dados relativos aos indivíduos do grupo B (placebo).

\begin{tabular}{|c|c|c|c|c|c|}
\hline Identificação & Idade (anos) & Peso (Kg) & Partos & EVA inicial & $\begin{array}{c}\text { EVA após a } \\
\text { TENS }\end{array}$ \\
\hline 01 & 24 & 70,5 & 1 & 5 & 0 \\
\hline 02 & 23 & 52 & 2 & 5 & 2 \\
\hline 03 & 20 & 57 & 1 & 7 & 7 \\
\hline 04 & 23 & 60 & 2 & 5 & 5 \\
\hline 05 & 19 & 50 & 1 & 5 & 5 \\
\hline 06 & 16 & 53 & 1 & 10 & 10 \\
\hline 07 & 22 & 52 & 1 & 4 & 4 \\
\hline 08 & 22 & 74 & 1 & 10 & 10 \\
\hline 09 & 35 & 60 & 2 & 9 & 9 \\
\hline 10 & 25 & 104 & 2 & 5 & 6 \\
\hline 11 & 23 & 48 & 2 & 5 & 5 \\
\hline 12 & 23 & 58 & 2 & 8 & 8 \\
\hline 13 & 21 & 77 & 2 & 8 & 9 \\
\hline 14 & 31 & 57 & 1 & 6 & 9 \\
\hline 15 & 25 & 80 & 1 & 10 & 10 \\
\hline
\end{tabular}

numéricos (0-10), a qual se constitui num método rápido, direto e prático de avaliação da dor, sem sofrer influência de barreiras lingüísticas e culturais, sem contar que os pacientes não ficam sobrecarregados em preencherem formulários extensos ou complexos, contendo múltiplas perguntas.

\section{CONCLUSÃO}

Levando em consideração as condições experimentais deste estudo, podemos concluir que a utilização da TENS pode constituir mais um recurso analgésico, bastante viável, pela simplicidade, praticidade e viabilidade econômica, para o controle da dor em mulheres submetidas à cesariana.

Entretanto, continua havendo a necessidade de investigações sistematizadas, especialmente através de estudos clínicos randomizados e controlados, que avaliem a efetividade isolada da TENS ou em associação a determinados fármacos, podendo ser aplicada também antecipadamente, durante e no pós-parto.

\section{REFERÊNCIAS BIBLIOGRÁFICAS}

1. Starkey C. Recursos terapêuticos em fisioterapia. $1^{\mathrm{a}}$ ed. Manole, São Paulo. 2001. P. 380-82.

2. Rushton DN. Electrical stimulation in the treatment of pain. Ann Disability and Rehabilitation 2002; 24 (8): 407-15.
3. Johnson MI. Transcutaneous electrical nerve stimulation (TENS) in the management of labour pain: the experience of over ten thousand women. Ann British Journal of Midwifery 1997; 5: 400-5.

4. Brosseau L, Milne S, Robinson V, Marchand S, Shea B, Wells G, et al. Efficacy of the transcutaneous electrical nerve stimulation for the treatment of chronic low back pain. Ann Spine 2002; 27 (6): 596-603.

5. Lianza S. Medicina de reabilitação. 3aㅡ ed. Guanabara Koogan. Rio de Janeiro. 2001. P. 200-4

6. Melzack R, Wall PD. Pain mechanisms: a new theory. Science Nov 1965; 150 (699): 971-9.

7. Greer KR, Hoyt JW. Pain: theory, anatomy, and physiology. Crit Care Clin. 1990 Apr; 6 (2): 227-34.

8. Ersek RA. Transcutaneous electrical neurostimulations: a new therapeutic modality for controlling pain. Clin Orthop Relat Res. 1997 Oct; (128): 314-24.

9. Hollinger JL. Transcutaneous electrical nerve stimulation after cesarean birth. Ann Phys Ther 1986; 66 (1): 36-8.

10. Smith CM, Guralnick MS, Gelfand MM, Jeans ME. The effects of transcutaneous electrical nerve stimulation on post-cesarean pain. Ann Pain 1996; 27 (2): 181-93.

11. Reynolds RA, Gladstone N, Ansari AH. Transcutaneous electrical nerve stimulation for reducing narcotic use after cesarean section. Ann J Reprod Med Nov 1987; 32 (11): 843-6. 
12. Lantsev EA, Smirnov AA. The use of transcutaneous electric neurostimulation for postoperative analgesia in parturients undergoing cesarian section. Ann Anesteziol Reanimatol 1990; 6: 66-9.

13. Navarro NC, Pacheco CM. Transcutaneous electric stimulation (TENS) to reduce pain after cesarean section. Ann Ginecol Obstet Mex 2000; 68: 60-3.

14. Polden M, Mantle J. Fisioterapia em ginecologia e obstetrícia. 2aㅡ ed. Santos. São Paulo. 1997. P. 200-10.

15. Ferreira CHJ, Payno SMA. Eletroestimulação nervosa transcutânea como recurso de alívio da dor no trabalho de parto. Femina 2002: 30 (2): 83-6.

16. Simkin P. The meaning of labor pain. Birth 2000 Dec; 27 (4): 254-5.
17. Niven CA, Murphy-Black T. Memory for labor pain: a review of the literature. Birth 2000 Dec; 27 (4): 244-53.

18. Van der Spank JT. Pain relief in labor by transcutaneous electrical nerve stimulation (TENS). Ann Arch Gynecol Obstet 2000; 264 (3): 131-136.

19. Mendoza MP, Villa Verde CA, Lopez FB, Villeges ST, Reyes E. Efectividad de la Estimulcion Electrica Transcutanea (TENS) en el alivio del Trabajo de Parto. Ann Rev Mex Anest 2000; 23 (2): 60-65.

20. Van der Ploeg JM. Transcutaneous nerve stimulation (TENS) during the first stage of labour: a randomized clinical trial. Ann Pain 1996; 68: 75-78. 